\title{
La nature du sujet dans les syntagmes prédicatifs en finnois suomi
}

Ces dernières années ont vu paraître dans les périodiques spécialisés de Finlande une série d'études plus intéressantes les unes que les autres portant sur l'interprétation des termes qui assument en finnois moderne le rôle de sujet d'un prédicat.

Il n'est pas dans notre intention de reprendre ici l'examen de ces exposés qui ont suscité une large controverse entre spécialistes, notamment dans les colonnes des revues telles que Virittäjä, Sananjalka et les Finnisch-ugrische Forschungen. Il nous a cependant paru qu'il ne serait pas sans utilité d'y ajouter quelques remarques générales et même quelques observations de détail instituées à partir d'un point de vue différent, celui d'un observateur dont la langue maternelle connaît l'expression de distinctions analogues sinon équivalentes. En effet, bien que le français ne présente pas de distinction morphologique pour exprimer le partiel opposé au total, il fait systematiquement et régulièrement usage de procédés syntactiques pour parvenir à la même fin. Cet état de choses n'a nullement échappé à l'attention des linguistes qui ont traité des faits finnois mais ils se sont bornés à n'y faire que de très sommaires allusions.

Notre propos est donc de dire comment le problème finnois se présente aux yeux d'un linguiste dont la langue maternelle est le français et en quoi l'état finnois lui paraît à la fois ressembler à l'état français et en même temps en différer. Par là il espère faire au moins en partie ressortir ce qu'il y a de spécifiquement original dans le comportement du finnois.

Comme on le sait. le sujet, quand il est exprimé en finnois 
par le moyen d'un nom (substantif ou pronom) peut se présenter sons deux aspects morphologiques différents: il peut être au cas nominatif ou all cas partitif.

Mais alors qu'en français, le sujet partiel et le sujet total sont rgaux en droit, c'est-à-dire capables d'assumer des fonctions identiques par rapport au rerbe et aux compléments de celuici, le sujet au nominatif et celui an partitif n'ont pas en finnois les mêmes aires d'emploi. Par "finnois", nous entendons naturellement ici la langue dite mormale» de l'État de Finlande, telle qu'elle fst enseignte officiellement et utilisée publiquement.

Ainsi qu il a été maintes fois exposé, le sujet partiel finnois, supporté par le cas partitif, ne se construit normalement ou "correctement" quavec un verbe intransitif de nature. Il ne s'accorde pas en nombre avec le verbe te ne saurait être combiné avec un attribut ou complément de prédicat que si celui-ci figure aux cas essif ou partitif. Le sujet partiel finnois est donc un sujet à fonctions limitées. Cette limitation se retrouve dans les parlers dialectaux et même en estonien où déjà Wiedemann avait fort clairement défini son emploi (Grammatik der Ehstnischen Sprache, pp 591-599). Il est vrai que Lauri Kettunen, entre autres, a relevé en vepse des constructions où le sujet partiel est associé à des verbes transitifs mais les exemples qu il a cités dans son remarquable ouvrage sur la syntaxe de cette langue (Vepsän murteiden lauseopillinen tutkimus, MSFOn 86, p 50) sont presque tous assez ambigus. D'une part le sujet y figure au pluriel de la 3-e personne et les verbes transitifs avec lesquels il est construit sont sans complément d'objet exprimé. Par contre, en suomi même, on rencontre sà et là des phrases où le verbe à sujet partiel comporte vraiment un complément d'objet, bien que de pareilles constructions soient condamnées par les grammairiens (voir en particulier: E. A. Saarimaa, Kielenopas, p 15051). Récemment encore, nous avons ln: Monta Iivanan ja Ofimjankin lasta oli saanut siellä lecon. (Elna Pelkonen: Karjalan meren äärellä, p 190). "Plus d'un enfant d'Ivan et aussi d'Ophémie y avait trouvé le repos."

Ce qu'il importe de retenir de tout cela, c'est que le sujet 
partiel finnois n'est pas un sujet à part entière. Il n'entre que dans des constructions où le verbe est intransitif et n'est meployé qu'au singulier.

Le problème qui se pose et qui revêt une grande importance pratique est de savoir en quoi consiste la différence de signification, du point de vue sémantique, entre les énonciations où le sujet est employé au partitif et celles où il est employé au nominatif. C'est à ce sujet que se sont précisément opposées les opinions des spécialistes au cours de la controverse à laquelle il a été fait allusion ci-dessus.

Comment les faits se présentent-ils? Nous avons d'abord à distinguer les phrases où le sujet partiel figure au singulier et celles où il est employé au pluriel, ces dernières étant de beaucoup les plus fréquentes. Il y a en outre à distinguer entre les phrases positives et les phrases négatives. Ces dernières sont également plus fréquentes quand il s'agit du sujet partiel an pluriel. Ies oppositions d'emploi qui en résultent sont les suivantes:

$A-$ Sujet partiel

1) an singulier positif

2) au pluriel positif

3) au singulier négatif

4) au pluriel négatif

$B$ - Opposition du sujet partiel et du sujet total.

Ce n'est pas tout. Il ne faut pas non plus négliger l'ordre des mots. Il n'est pas indifférent que le sujet précède ou suive le verbe. Wiedemann avait déjà constaté, par exemple, que les cas où le sujet partiel suivait le verbe étaient, dans l'estonien de son temps, de beaucoup les plus fréquents.

Il ne saurait être question d'aligner ici des files interminables d'exemples pour illustrer ces faits déjà bien connus et bien analysés. Nous nous bornerons à mentionner quelques exemples qui nous ont paru typiques, relevés au hasard dans le dépouillement auquel nous avons procédé depuis de nombreuses années (depuis 1940 plus précisément) et qui se montent à 
plusieurs milliers. Ceux que nous allons utiliser dans ce qui suit ont été empruntés de préférence à des textes relativement récents.

Le classement numérique de ces emplois impose une prenière observation: l'emploi du sujet partiel au pluriel est d'une fréquence sensiblement égale chez tous les auteurs. Il s'agit là d'un procédé vraiment bien installé dans la langue alors que l'emploi du sujet partiel au singulier, plus particulièrement dans les énonciations positives, est plus ou moins utilisé selon les auteurs. Certains écrivains y recourent volontiers, d'autres en revanche semblent l'éviter, sauf dans quelques rares constructions apparemment stéréotypées.

Comme tous les auteurs l'ont signalé et comme le professeur Aarni Penttilä y a particulièrement insisté dans sa grammaire où il a fourni une liste très longue à ce propos, le sujet partiel apparaît surtout en combinaison avec certains verbes. (Suomen kielioppi, pp 623-627). Il est donc de bonne méthode, pour discerner la différence de sens des constructions en présence, de choisir des phrases où figure un même verbe. Prenons par exemple le verbe kuulua "se faire entendre, s'entendre". Nous allons nous efforcer de retrouver des phrases pour ainsi dire parallèles afin que la seule variable soit le sujet. Nous lisons ainsi:

Silloin ei enää haukuntaa kuulunut, kuului vain hïhtomme aiheuttama kova hahina, meluten erämaan syvässä hiljaisuudessa. (E. A. Järvinen: Ihmisiä suurilla selkosilla, p 68).

Nous traduirons cette phrase comme suit, car la traduction est importante pour faire ressortir l'originalité du procédé finnois:

"On n'entendit plus alors d'aboiement, on entendait seulement le dur crissement produit par nos skis tonitruant dans le profond silence de la nature vierge."

Nous lisons ensuite (id, p 125):

Mutta haukuntaa ei kuulu, kuuluu vain sukisien kahinaa niiden liukuessa rivannekelissä yli jäisten, aamunvalossa säihkyvien hankien.

"Mais on n'entend plus d'aboiement, on entend seulement le crissement des skis tandis qu'ils glissent sur la neige glacée 
par dessus les étendues de neige gelée qui étincellent dans la lumière du matin.»

On aura remarqué que nous n'avons pas rendu en français le partitif kahinaa par "du crissement" qui en serait la transposition littérale. Nous avons employé l'article défini et cette divergence suffit à dénoncer déjà que l'usage finnois ne répond pas ici à l'usage français. Les deux énoncés considérés sont presque synonymes dans leur intégralité. Pourquoi là le nominatif et ici le partitif? Quelle nuance de sens l'auteur a-t-il voulu exprimer par cette opposition?

Poursuivons notre examen. Soient deux phrases moins complètement parallèles mais qui se rapportent toutes deux à des situations comparables, extraites l'une et l'autre d'un même ouvrage afin d'opérer dans l'unité du stỵle, autant que possible. Nous lisons cette fois:

Ulkoa kuului yksinkertaisten ikkunoiden läritse jatkuva pehmeä suhina ja aivan pientä lipinää kun vesi putosi katolta sireeninlehtiin. (Annikki Sankari: - ettei teitä tuomittaisi, p 110)

"De dehors, on entendait à travers les fenêtres simples un bruissement doux continu et un tout petit susurrement tandis que l'eau tombait du toit sur les feuilles des lilas."

Hiljan kasvot kuumenivat ja korvissa tuntui ilkeää suhinaa. (id, p 156)

"Le visage d'Hilja s'échauffa et il lui sembla entendre dans les oreilles un bruissement mauvais.»

Il est curieux de constater que dans la première phrase citée, le même verbe kuulua a deux sujets, l'un au nominatif singulier (suhina) et l'autre au partitif singulier (lipinää).

Si l'on confronte maintenant les quatre exemples dont nous venons de donner la traduction française, on est bien forcé de se rendre à cette évidence que l'emploi de la distinction formelle du partitif et du nominatif ne répond nullement à l'usage français du partiel opposé au total mais que la distinction símantique qui se dégage de l'opposition relevée en finnois est d'une autre nature. Le partitif n'indique pas du tout la partialité mais bien l'embarras où se trouve l'auteur pour définir avec précision le concept qu'il tient à exprimer. C'est bien ce que senble confirmer une phrase telle que: 
Kun tarktiaan kunnteli. kuului kosken kohina. (Järvinen, op.cit. p 88)

"Quand on écoutait attentivement, on entendait le mugissement du rapide."

L'observateur a ajusté son ouie et sa perception en est devenue précise. Le nominatif kohina dénonce que le bruit est identifié sans aucune hésitation possible. Au contraire, si l'observateur ne peut déterminer avec précision la nature du bruit qui lui parvient, il emploie le partitif:

Pimeästä metsästä, ei kovin kaukaa, kuuluu kuin pauketta, valtavaa pauketta, ja sitten kohinaa. (id, p 11)

"De la forêt obscure, pas de très loin, se fait entendre comme une détonation, une puissante détonation et ensuite un mugissement.»

Nous serions même tenté d'écrire en français »une sorte de mugissement", c'est-à-dire quelque chose qui participe du mugissement sans qu'on l'identifie sûrement comme tel.

Ainsi donc, dans les cas analysés ci-dessus, l'opposition morphologique partitif / nominatif a été interprétée comme une distinction entre la perception nette et la perception indistincte ou d'identification incertaine. Avons-nous affaire à une distinction déterminé / indéterminé? Ce serait s'avancer beaucoup que de l'affirmer. Ce que le partitif implique, c'est une affirmation moins nette, une expression d'un contour moins clairement dessiné ou, si l'on veut, moins fermé.

Monsieur Vilho Kallioinen, interrogé sur ces exemples a bien voulu me confier que le partitif évoque ì son esprit une notion de durée. Les bruits désignés par des substantifs au partitif sont d'après lui des bruits qui se prolongent durant un temps indéterminé alors que ceux exprimés par des nominatifs sont des bruits enregistrés comme tels mais sans qu'ils soient conçus comme affectés d'une durée plus ou moins prolongée. Prenons bonne note de cette importante observation et poursuivons notre enquête: Nous lisons:

Nousen pystyyn ja tempaan pyssymi, samalla tähyilen, näkyykö lioiraa. (Järvinen, op.cit. p 170)

"Je me mets debont et je saisis mon fusil tout en guettant pour voir si le chien s'aperçoit." 
Le mot koiraa est an partitif et nous devrions traduire en français, pour bien refliter l'expression finnoise: "si quelque chose comme un chien saperçoit” ou encore: "si quelque chose qui ressemble au chien s'aperçoit». Mais dès qu'il a aperçu l'animal, le chasseur s'ficrie:

Tuossa on koira. (id, p 171)

"Le chien est là-bas" (= "Il est là-bas, le chien") et cette fois le mot loira est an nominatif. C'est qu'il n'y a plus ni doute ni indítermination. Que notre explication n'est pas tendancieuse. c'est ce rue corrobore cet autre exemple extrait dı même ouvrage dı même auteur (p 24):

Hänen mielessään liyllä liäväisee, että pitäisi vieläkin tehdä kierros pajupensaikossa, tutkia, näkyisikö tulisijaa...

"Il lui passe sans doute par l'esprit qu'il faudrait faire encore un tour dans les taillis de saules pour examiner si on ne verrait pas l'emplacement d'un feu ...."

Le chasseur est ici a la recherche du moindre indice susceptible de déceler s'il a été fait du feu et c'est ce que veut dire le partitif.

Passons à un autre texte. Nous lisons (Annikki Sinkarı. op.cit. p 37):

Sieltä tuli mutkaa ja pitempi matha, mutta vaija ei halunnut mennä lypsypailian ohitse.

"Par là, ça faisait un détour et un trajet plus long Inais le vieux ne désirait pas passer par l'endroit où l'on trayait."

Le mot mutl:aa winuosité, crochet, détour" est au partitif alors que lo mot matka strajet, itinéraire» est au nominatif. C'est que la nature du détour à faire est présentée comme plus ou moins indéterminée alors que le trajet à parcourir devient nécessairement plus long. Cela suggère que le détour à faire est moins nettement présent à l'esprit du personnage mais que ce qui est par contre tout à fait net dans sa représentation. c'est la longueur plus grande du trajet qui en résultera. Le premier concept est de contour mal arrêté alor's que le second se présente avec précision. M. Vilho Kallioinen a bien voulu me dire qu'il percoit comme une notion de durée prolongée dans le partitif.

Cette opposition peut, naturellement se refléter parfois en 
français par l'opposition partiel / total comme dans le cas suivant:

Näetkö, tuossa leijailee savua? - Todellakin, siinä on savua.

"Est-ce que tu le vois? Il y a là-bas de la fumée qui flotte? Oui, vraiment, il y a de la fumée.»

qui s'oppose à:

Sieltä täältä kohoaa $s$ a v u, jonkin korsun piipusta, ja jossakin hyrisee auto. (Järvinen, op.cit. p 33)

"Ca et là s'élève une fumée de la cheminée d'une cabane et quelque part vrombit une auto."

Ce qui nous rappelle que le nominatif finnois sert à individualiser la perception alors que le partitif lui confère un contour indistinct. Mais cette distinction n'a rien à voir avec la spécification proprement dite, le nominatif finnois répondant ici à l'indéfini français exprimé au moyen de l'article indéfini "un", alors que dans un autre cas analogue, le français répond par l'article défini:

Lumi ei nyt ainoastaan upota, vaan sen alle on kerääntynyt v e $t$ ta. Kun polkaisee lumeen, tulee $v$ e s $i$ esille. (id, p 214)

"La neige n'enfonce pas seulement mais sous elle s'est accumulée de l'eau. Quand on piétine dans la neige, l'eau vient en surface."

Il résulte de ces analyses que l'opposition morphologique finnoise est exploitée de telle façon que les constructions où elle joue ne correspondent nullement à celles, où le français tire parti de l'opposition du partiel et du total d'une part, du défini et de l'indéfini d'autre part. Les deux systèmes ne se rencontrent que dans des cas isolés.

Voyons maintenant en quoi consiste l'opposition d'emploi du sujet au partitif singulier et du sujet au partitif pluriel. Nous lisons par exemple:

Jo aamuvarhaisesta ajoi kylään hevosia. Tuli ukkoa, urosta ja nuorta poikaa, emäntää ja koreata tyttöä pitäjän kaikilta kulmilta. (Elna Pelkonen: Karjalan meren äärellä, p 124)

"Depuis le matin de bonne heure des (voitures à) chevaux arrivaient au village. Il venait des hommes, des gars et des jeunes gens, des matrones et de jolies filles de tous les coins du canton.» 
Le sujet du premier verbe est hevosia »des chevaux» (en réalité "des voitures à chevaux») qui est un partitif pluriel alors que les sujets qui viennent après le verbe de la seconde proposition sont des partitifs singuliers bien qu'ils s'appliquent à des personnes et non à des masses plus ou moins indivisibles. Or qui ne sent que ces partitifs sont d'acception emphatique? Leur emploi fait ressortir le nombre considérable des gens qui viennent au village de toutes parts. Dans ce cas, le français présente un emploi correspondant du partitif singulier opposé au pluriel:

$$
\begin{aligned}
& \text { Il y a des voitures sur la route } \\
& \text { Il y a de la voiture sur la route, }
\end{aligned}
$$

la seconde énonciation suggérant que les voitures perçues sur la route sont en nombre inusité. C'est que le partitif pluriel finnois a presque la même valeur sémantique sinon syntaxique que le partitif pluriel français:

Jono pysähtyi heti, ja käs $i \ddot{a}$ kohoili. (Järvinen, op.cit. p 185)

"Le cortège s'arrêta aussitôt et des mains se levèrent."

Le partitif pluriel käsiä "des mains" est ici exactement superposable à la construction française par laquelle nous l'avons transposé. On a de même (id, p 13):

Kun jälkiä näkyi vain yhdessä pälvessä, oli karhun seuraaminen mahdotonta...

"Comme des traces ne s'apercevaient qu'en un seul endroit sans neige, il était impossible de suivre l'ours..." Ici encore jälkiä, partitif pluriel, peut être rendu par »des traces».

Naturellement, il arrive que le partitif finnois fasse partie, en tant que sujet au pluriel, de constructions où il entre en combinaison avec des termes qui, en français, ne sauraient se combiner avec l'emploi du partitif, même au pluriel. Nous lisons ainsi (id, p 169):

Siellä näkyy taivasta vasten pitkien kuusten latvoja.

"Là s'aperçoivent contre le ciel les cimes de hauts sapins."

Nous avons apparemment rendu latvoja, partitif pluriel par "les cimes" où l'article défini exclut toute notion de partitif mais par contre, cette même notion se trouve exprimée dans 
"de hauts sapins" alors que le génitif pluriel finnois est indifférent ici et en saurait pour cette raison exprimer la partialité. La divergence est plus apparente que réelle. La notion de partialité a été transférée des "cimes» sur les »sapins». Sans doute, nous pourrions dire à la rigueur en français »des cimes de hauts sapins» mais cette construction semblerait insolite parce que pléonastique.

Des exemples ci-dessus, il résulte que l'emploi du partitif pluriel du finnois correspond en gros à celui du français, mis à part les quelques cas où, pour des raisons de structure, les deux langues divergent nécessairement. En revanche, le sujet partiel au singulier joue en finnois un rôle différent de celui assumé en français par la construction correspondante avec l'article partitif. Cette différence, elle n'apparaît pas non plus dans tous les cas d'emploi. Il y a des énonciations où le français et le finnois vont de pair comme cela a été depuis longtemps signalé. Quand on lit en finnois:

minulla oli o n ne a (Järvinen, op.cit., p 35), on ne peut que traduire en français:

"j’ai ell de la chance"

De même, le partitif finnois répond au partitif français dans:

Korpi-Santeri sanoo, että siztä sttaa aina, jos vain on lo hta joessa. (id, p 61)

"Alexandre des Bois dit qu'on les y prend toujours, pourvu qu'il y ait du saumon dans la rivière».

Ise mot lohta, partitif singulier de lohi "saumon", se rend naturellement par »du saumon» car, dans la même circonstance, nous autres Français, nous employons le partitif. Par contre, dans trop d'autres cas, le partitif finnois ne saurait être traduit par le partitif singulier français:

Täällä on sitä, mitä ei muralla ole. On erämaan la a $j$ u u $t$ t a ja erämaan ra uha a. (id, p 12)

"Il y a ici ce qu'il n'y a pas ailleurs: il y a l'étendue de la nature vierge et la paix de la nature viergen.

LE finnois dit "de l'étendue» et aussi "de la paix» sous-entendant par là que le paysage où se trouve l'auteur participe à la vaste étendue et à la grande paix des espaces vierges. 
L'opposition devient même encore plus flagrante quand elle est contenue dans une seule et même phrase:

Tuli vaikeita päiviä, jännittävää odotusta. (id, p 230)

"Vinrent des jours difficiles, une attente tendue." Pour rendre le concept exprimé par le finnois, le traducteur français devrait alors transposer en:

"Vinrent des jours difficiles, d'attente tendue».

Quelle interprétation faut-il donner à ce désaccord qui jure avec l'accord enregistré dans les autres cas? Celle-ci: le finnois s'est trouvé, comme le français, en possession de deux tournures pour exprimer le sujet. Il a tiré parti de ce dualisme en s'efforçant de spécialiser chaque tournure dans des acceptions particulières. Mais comme les deux idiomes, qui ne sont ni parents ni voisins, ont cheminé chacun séparément, la spécialisation obtenue de part et d'autre ne peut coïncider. Sémantiquement, le point de départ était le même: un procédé d'expression de l'ablatif. En finnois, ce procédé consiste en l'affixation d'un morphème sous les espèces du suffixe -ta / -tä qui a été originellement le suffixe du cas ablatif. En français, il s'agit de la préposition de, héritée du latin où, construite avec l'ablatif, elle exprimait la notion d'ablatif. Les cheminements français et finnois ont produit des parallélismes sémantiques dans plusieurs cas, notamment au pluriel et, au contraire, dans d'autres cas, particulièrement au singulier, le finnois a tiré de l'emploi du partitif en fonction de sujet un parti à la fois plus fréquent et plus complexe que le français. Cela s'explique par bien des raisons et notamment par celle-ci: le français dispose d'un jeu d'articles alors que le finnois n'a pas réussi à en développer un en dépit de plusieurs velléités constatées au cours de son histoire.

Plusieurs théoriciens finlandais ont fait valoir, non sans apparence de raison, que les syntagmes prédicatifs à sujet au partitif étaient essentiellement des constats d'existence. C'est ainsi que s'interpréterait une opposition comme celle-ci:

. . aika ajoin kuului kollietta ja helinää. (Järvinen, id, $\mathrm{p} 45$ )

... »de temps en temps, un choc, un cliquetis se faisaient entendres

et: 
Tuuli tohisee ja sade valuu, rapisee ikkunaruutua vasten, ka $l k u t u s$ ja helinä kuuluu. (ibid)

"Le vent mugit, la pluie tombe à verse, frappe contre la vitre, un choc et un cliquetis se font entendre."

Dans la première énonciation, il s'agit d'un constat. Dans la seconde, l'assertion concerne les bruits qui ont déjà été précédemment perçus. Leur existence a été enregistrée, il n'y est plus fait qu'allusion. Le nominatif renvoie pour ainsi dire au premier constat qui est exprimé sous les espèces du partiti.

Mais cette interprétation, si valable qu'elle soit dans de nombreux cas, n'est pas toujours soutenable. Il arrive que le nominatif soit bel et bien employé pour exprimer un constat d'existence:

Loiske loittoni ja heikkeni, mutta ennenkuin se vaikeni kokonaan, alkoi samalta suunnalta kuulua kiihkë̈ hauk $u$ n $t a . "$ (id, 121)

"Le clapotis s'éloigna et s'affaiblit mais arant qu'il se tût complètement, un furieux aboiement commença à se faire entendre dans la même direction."

Nous sommes bien en présence d'un constat qui révèle l'existence de ce nouveau bruit succédant à l'autre en train de s'évanouir et, pourtant, nous avons un sujet au nominatif.

Inversement, nous lisons (Toivo Pekkanen: Lapsuuteni, p 235)

Yöllä heräsimme jälleen ammuntaan, jota kesti tänä yönä kauemmin kuin edellisenä.

"La nuit, nous nous réveillâmes à nouveau au bruit de la fusillade qui dura cette nuit-ci plus longtemps que la précédente."

Or le même écrivain avait exprimé une idée voisine dans un passage précédent du même ouvrage (p 146):

... ja vaikka yritin juosta voimieni mukaan, matka kesti kauemmin kuin koskaan ennen.

". . . et quoique je m'évertuai à courir de toutes mes forces, le trajet dura plus longtemps que jamais aupararant."

Ni l'une ni l'autre de ces deux phrase ne comporte de constat d'existence. Le concept de tir ou celui de trajet sont des don- 
nées connues du lecteur et cependant, dans le premier cas, nous avons un sujet au partitif (jota, relatif "quip) et dans l'autre un nominatif (matka strajet, chemin"). A quoi vise alors la distinction?

Il semble bien que les théoriciens aient eu tort, qui ont voulu délimiter trop précisément les aires d'emploi du partitif par opposition à l'emploi du nominatif. Le sujet parlant, ayant conscience qu'il peut se servir alternativement de deux formules, essaie d'employer l'une plutôt que l'autre pour obtenir des nuances plus ou moins subtiles et plus ou moins variables selon les cas. Ce qui le confirme, c'est l'embarras où se trouve l'informateur de langue finnoise lorsqu'on lui demande à brûlepourpoint ce que signifie exactement l'emploi du partitif singulier dans telle ou telle phrase donnée. Il accorde qu'une nuance doit exister mais il ne sait pas toujours la définir. Rien n'est plus instructif à cet égard que de passer d'un informateur à l'autre et de solliciter l'interprétation de la même phrase. Des explications contradictoires sont souvent apportées qui n'éclairent naturellement pas l'étranger sur la signification exacte de la phrase incriminée.

Ce qui vient encore compliquer les choses, c'est que le sujet parlant, de par l'enseignement scolaire qu'il a reçu, porte en lui cette notion que le partitif est le cas qui exprime la partie d'un tout. Il tend donc à interpréter tout partitif dans ce sens. On y rattache tout ce qui évoque une participation même lointaine à un tout:

Lehtien alla oli musta a... (Eeva Kilpi: Noidanlutiko, p 130)

"Sous les feuilles, il faisait noir (= il y avait du noir)..." Pihamäntyjen alla oli jo $h \ddot{a} m \ddot{a} r a ̈ a ̈$. (Ilmari Saarinen: Unennäkijät, p 5)

"Sous les pins de la cour, il faisait déjà sombre."

- Katsokaa, kuinka pime ä̈a on ulkona, sanoi Eeva. (id, p 22)

"Regarde comme il fait nuit dehors, dit Eva."

On pourrait multiplier les citations de ce type où le sujet figure au partitif singulier pour exprimer la présence d'un phénomène naturel dont il est malaisé de se représenter qu'il 
peut être conçu comme la partie d'un tout plus ou moins indivisible. On lit encore:

On sytän hiljaista. (Järvinen, op.cit. p 7)

"Il y a un profond silence."

Vielä on kuitenkin viil e ät ä... (id, p 37)

"Il fait pourtant encore frais..."

Mais il y a lieu de se demander ici dans quelle mesure le recours au partitif n'est pas suggéré par le besoin d'éviter quelque gênante ambiguité. Le nominatif pourrait en effet s'interpréter parfois comme un attribut d'un sujet implicite de 3-e personne du singulier, nous voulons dire inexprimé par le pronom. Il s'agirait alors d'un expédient purement formel qui n'impliquerait nullement une catégorie sémantique particulière.

C'est aussi ce que suggère d'autre part l'emploi de clichés qui se retrouvent partout. Ainsi, avec le mot väki "gens, peuple" et plusieurs rerbes signifiant le rassemblement, la pénétration, l'afflux, etc, on retrouve d'un texte à l'autre le sujet employé au partitif singulier (väkeä):

Sitä mukaa kuin huoneeseen kertyi $v \ddot{a} k$ e ä, vaimeni tarinointi... (Elna Pelkonen: Karjalan meren äärellä, p 196)

"A mesure que les gens se rassemblaient dans la pièce, les récits se taisaient ..."

Iltahämärissä alkoi väke ä kerääntyä lähistölle. (Väinö Linna: Täällä pohjantähden alla, p 315)

"Au crépuscule, des gens commencèrent à s'attrouper dans le voisinage."

Lisäksi sopi väk e ä sen vieressä oleviin kamariin ja keittiöön. (ibid)

"En outre, des gens trouvèrent place dans la chambre et la cuisine attenantes.»

... juoksipa joissakin mökeissä $v \ddot{a} k$ e ä rappusillekin (id, p 313)

... mil y avait même des gens qui couraient dans quelques masures jusque sur les escaliers.»

Il est difficile de se défendre du sentiment que ce sont là des formules stéréotypées que le sujet palrant emploie automati- 
quement sans y impliquer de lui-même une nuance particulière quelconque.

Par ailleurs, certaines tournures qui apparaissent çà et là amènent à penser que le recours conscient au partitif, surtout au partitif singulier est souvent le résultat d'une recherche délibérée du locuteur ou de l'écrivain. Ainsi, nous lisons:

Koti miltei jo näkyy. (Järvinen: op.cit. p 151)

"La maison s'aperçoit déjà presque."

où le nominatif singulier se trouve employé pour indiquer pourtant une vision incomplète alors que les théoriciens qui ont traité de ce problème ont constamment cité des constructions telles que:

Kirkko a näkyy puiden oksien rälistä.

"L'église s'entrevoit à travers les branches des arbres" (cp en dernier lieu Paavo Siro: Sananjalka II, p 38, etc).

Ces dernières constructions (au demeurant rarissimes) auraient pour fonction d'exprimer la perception incomplète. I.e partitif indiquerait que l'objet dont l'existence est constaté ne serait que partiellement reconnu ou identifié. Mais cette explication cadre-t-elle avec les faits?

Ne se trouve-t-on pas devant un effet de style, fruit d'une recherche consciente de la part du locuteur? Il sait qu'il peut utiliser le partitif en fonction de sujet, il n'ignore pas que l'emploi habituel est, dans un cas pareil, celui du nominatif; il met alors en valeur le sujet du seul fait qu'il l'emploie au cas inattendu. Est-il nécessaire d'inventer des classifications sémantiques pour rendre compte de ce phénomène général? Nous ne le pensons pas et nous estimons vain de vouloir à tout prix ranger tel emploi du partitif sujet dans la catégorie du partiel, tel autre dans celle de l'indéfini ou de l'indéterminé. Ce qui est sûr, c'est que le locuteur de langue finnoise a conscience qu'il dispose de deux constructions quand il veut exprimer une assertion finie. L'une comporte le sujet au nominatif et l'autre le sujet au partitif. En gros, il est admis que le partitif implique la notion de partialité alors que le nominatif signifie la totalité. A partir de ces deux notions fondamentales, le locuteur tire parti des deux formules au gré des circonstances. 
Nous avons lu ci-dessus que le verbe kuulu- se construit volontiers avec un sujet au partitif et nous avons analysé plusieurs exemples de cet emploi mais nous avons constaté par là même que le nominatif apparaît également sans que la nature de la notion en cause (le genre de bruit ou de sonorité) explique le recours au partitif plutôt qu'au nominatif ou inversement. Ce qui paraît avoir décidé le locuteur, ici l'écrivain. c'est le genre de perception auquel il veut faire allusion. S'il veut nous faire saisir que le bruit perçu est parvenu à ses oreilles comme une entité bien nettement circonscrite, il a employé le nominatif, dans le cas contraire, dès que le bruit n'est plus cerné dans un contour précis, il est exprimé au moyen du partitif. Nous en revenons donc ainsi à la grande opposition de départ: total / partiel. Si l'on en pouvait douter, il suffirait de s'arrêter devant cet exemple:

Kuhinan seasta kuului yhä Hellbergin ja Salinin vä $i t t e-$ l y ä ... (Väinö Linna: Täällä pohjantähden alla, p 321)

"Au milieu du brouhaha, on entendait toujours la discussion de Hellberg et de Salin ..."

Le mot väittely "discussion" est au partitif singulier comme sujet de kuului "se faisait entendre" parce que dans le bruit des gens en train de prendre place dans la salle, cette discussion ne peut s'entendre ni distinctement ni complètement, que d'autre part, elle se poursuit toujours.

Il faut alors donner raison à Monsieur Paavo Siro quand il insiste sur ce trait essentiel de l'opposition des emplois du nominatif et du partitif.

Mais il est un dernier trait dans l'emploi du partitif sujet qui a également retenu l'attention de tous les chercheurs, c'est que le sujet apparaît nécessairement au partitif quand il supporte la négation.

On lit ainsi:

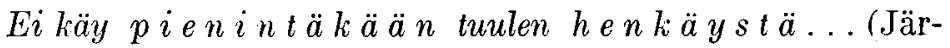
vinen, op.cit. $p 41$ )

"Il n'y a pas le plus petit souffle de vent ..." où le sujet de läy "va» est évidemment le partitif henkäystä "du souffle». Littéralement nous devrions dire en français pour nous plier au schéma de l'énoncé finnois: "Du plus petit souffle de vent 
ne va pas même pas..." Il suffit de produire cette phrase qui n'a rien de français pour faire apparaître la disparité qui existe ici entre l'usage finnois et l'usage français quand bien même il y a des cas où les deux usages concordent:

Savun haj и a ei kuitenkaan tunnu ilmassa. (id, 41)

"D'odeur de fumée, on n'en sent pas (il ne s'en sent pas) dans l'air."

Il en serait encore de même dans le cas (op.cit. p 40) de:

Ei edes kaukaa kuulu auton $h$ y $r i$ n $\ddot{a} \ddot{a}$ eikä la $u k a u k$ sia.

"On n'entend même pas au loin de vrombissement d'auto ni de détonations."

Toutefois, on aura constaté que dans nos traductions, nous avons "tourné» l'expression finnoise en français de façon à exprimer par un complément d'objet au partitif ce qui est en finnois un sujet au partitif. Nous nous trouvons donc devant une nouvelle discordance entre les faits finnois et les équivalents français par lesquels nous les avons interprétés. Il en serait de même dans les énoncés du type suivant:

Laurin is ä ä ei ollut näkynyt saunassa. (Ilmari Saarinen: Unennäkijät, p 49)

"On n'avait pas vu le père de Lauri dans la sauna."

Odotettiin tunti, toinen. Mutta is ä ä vain ei kuulumut. (id, 130)

"On attendit une heure, une autre (heure) mais toujours pas de nouvelles du père."

L'emploi du partitif en tant que sujet négatif a donc pris en finnois une extension comparable à celle qui est observée. par exemple, en russe. Cette négation du sujet peut même affecter des noms propres:

IIutta Al pia ei näy ei liuulu. (Järvinen, op.cit. p 196) "Mais on ne voit ni entend Alpi."

L'emploi du sujet au partitif quand la négation s'y réfère n'est pourtant pas d'une obligation absolue. Nous lisons ainsi:

Lun hän saapuu läheisen jängän reunalle, mihin ei ole $p i t k \ddot{a}$ $m$ a $t k a$, hän hämmästyy. (Järvinen, op.cit. p 215)

"Quand il arrive au bord du marais proche, jusqu'où il n'y a pas un long trajet, il est stupéfait.» 
(In aurait attendu le partitif mathaa alor's que l'on a affaire au nominatif.

Qu'il ne s'agit pas d'une erreur de typographie mais d'un emploi conscient de l'auteur, c'est ce que confirme l'énoncé:

- Nyt meillä ei ole enää pitk ä ma $t k a$. (id, p 19)

- "Maintenant, nous n avons plus un long chemin."

Ici encore matka est au nominatif. Ce nominatif surprend d'autant plus qu'il est construit avec le mot enää qui implique volontiers une idée de partialité! L'auteur a donc eu une intention en se servant du nominatif. C'est ce que corrobore cet autre exemple, relevé dans un autre ouvrage d'un autre auteur (Ilmari Saarinen: Unennäkijät, p 131):

Ei ole nyt a ik a tuumailla ja istua sormi suussa.

"Ce n'est pas le moment de réfléchir et de rester assis les bras croisés (littéralement: 'les doigts dans la bouche').»

Ce dernier emploi est plus clair. L'auteur a pensé au mot aika dans l'acception de "moment, occasion" et non dans celle de "durée indéterminée». Il s'agit donc d'un concept aux contours bien arrêtés. Or nous avons vu plus haut, dans les énoncés positifs que nous avons mentionnés, que le nominatif servait à l'expression de concepts arrêtés, précis alors que le partitif évoquait le vague, le flou, l'imprécis.

On se tromperait toutefois si l'on pensait que le partitif supporte en même temps l'expression de la catégorie de l'indéfini opposée à celle du défini. En effet, nous lisons dans Lapsuuteni "Mon enfance", de Toivo Pekkanen (p 231):

A m m u n ta a ei kestänyt kauan.

"Le tir ne dura pas longtemps"

où le mot ammuntaa est au partitif alors qu'il ne peut s'agir que d'un concept défini. En se reportant au contexte, on se persuade que le concept de tir a déjà été précédemment évoqué. Il s'agit d'une notion déjà connue, donc définie, ce qui n'empêche pas que l'écrivain ait employé le partitif pour l'exprimer. Cet emploi attire l'attention sur le mot ammunta et le met en valeur, il est donc emphatique et nous retrouvons une fois encore l'acception emphatique si frappante dans l'emploi du partitif singulier en tant que sujet. 
Le même auteur, dans une circonstance exactement analogue (op.cit. p 122) a également écrit:

$K i$ ih ty m ! s tä lesti ehlä noin pari tuntia.. .

"Liagitation dura peut-être dans les deux heures .... Ici aussi. il s'agit d'une notion qui a été antérieurement définie, de telle sorte que l'emploi du partitif ne saurait être interprété comme un procidé pour signaler lexistence du sentiment éprouvé par le héros. Seulement le partitif suggère que cette agitation a été d'une grande importance ou a joué un grand role. Nous avons affaire à un partitif emphatique, tout comme dans le cas de cette transfuge qui s"écrie (Järvinen, op.cit. p 28):

Yksin tulin, harhailin metsiä, satoja kilometrejä . . oli siinä tulemista.

"Je suis venue seule, jai erré dans les forêts, des centaines de kilomètres, quelle affaire que de venir!)

Il reste à déterminer en quoi consiste le sens de cette emphase. Il semble bien que cela soit rariable d'un cas à l'autre. Tantôt, c'est laspect quantitatif qui est mis en valeur, la dimension, l'intensité mais aussi la durée, ainsi qu'il ressort d'un exemple comme celui-ci (Toivo Pekkanen: Lapsuuteni, p 235):

Sillä ailiaa kun tarkast us ta jatkui...

"Pendant le temps que durait la perquisition..." oil le partitif précise l'aspect du verbe et lui confère la valeur de notre imparfait, acception qui est soulignée par le complément de durie sillä aikaa "pendant le temps que...".

Les théoriciens finnois n'ont guère par contre retenu parmi les cas d'emploi qu ils ont traité une tournure où pourtant le partitif singulier est actuellement d'une très grande fréquence. Nous faisons allusion aux formules où il est question d'un phénomène naturel:

Yhä sataa ulkona ja on $p i$ me ë̈̈̈. (Järvinen, op.cit. p 46) "Il plent toujours et dehors il fait obscur."

Siellä oli pime ä ä, mutta huomasin kuitenkin koivujen lomassa suuren lithluran hahmon. (id, 120)

"Il faisait sombre là, mais je remarquai pourtant entre les bouleaux une grande silhouette qui bougeait."

On pourrait se demander s'il ne s'agit pas de clichés où le 
partitif apparaît pour ainsi dire automatiquement. Il n'en est rien. On lit à côté:

On miltei pi me ä kuin syksyllä, ja ulkona sataa ja tuulee. (id, p 44)

"Il fait presque obscur comme en automne et dehors il pleut et il vente."

Ou encore:

Hetken on niin $p$ i me ä, ettei erota muuta kuin tulen ystävällisen puhelun. (id, p 210)

"Un instant il fait si obscur qu'on ne distingue rien d'autr" que l'amical bavardage du feu.»

Les situations sont comparables d'un cas à l'autre. Pourquoi tantôt le partitif et tantôt le nominatif?

Il semble bien que cette fois encore le partitif ait une valeur emphatique, ainsi que le suggère l'exemple suirant:

Katsokaa, kuinka $p i$ me ë̈̈ on ulkona, sanoi Eeva. (llmari Saarinen, Unennäkijät, p 22).

"Regardez comme il fait sombre dehor's, dit Eva."

Parcontre, il n'est pas douteux que certains mots soient systématiquement employés au partitif, d'autres au nominatif. C'est ainsi que nous n'avons pas relevé d'exemple de nominatif du mot hiljainen "silencieux" mais seulement le partitif dans les énonciations du type:

Hetken on hiljaista. (Järvinen, op.cit. p 187)

"Un instant, c'est le silence."

Ceci amène à rappeler que la nature même du mot qui sert de sujet intervient dans le choix que fait le sujet parlant du partitif ou du nominatif. Les mots yö "muit", ilta "soir" figurent toujours au nominatif:

On yö, muntamia päiviä ennen juhannusta. (Järvinen, op.cit. p 60)

"C'est la nuit, quelques jours avant la Saint-Jean."

Oli ilta, muistaaliseni toukokuu jo kulumassa. (id, p 119)

"C'était le soir, autant que je me rappelle déjà dans le courant de mai."

Cet emploi du partitif est d'autant plus frappant qu“il s'oppose parfois dans la même énonciation à celui du nominatif dans les mêmes fonctions: 
On hiostaran $k$ u u ma ja ympäristössä hi l ja is ta kuin joskus entisinä aikoina. (id, p 40)

"Il faisait chand à suer et dans les environs, c'était le silence comme dans les temps anciens."

Il n'est pas moins caractéristique de constater d'autre part quavec le verbe kuulua "se faire entendre", on ne rencontre jamais an partitif singulier les substantifs déverbatifs en -ahdus, -ähdys:

Ramnasta kuului liikettä, molskahduksia ja kolahdus. (Annikki Sankari: — ettei teitä tuomittaisi, p 30)

"Depuis la rive, on entendit du mouvement, des ploufs et un choc."

... sieltä kuului molskahdus ja sitten sadattelun tapaista. (ibid)

". . . de là, on entendit un plouf et quelque chose comme des jurons."

Or, on le sait, les dérivés onomatopéiques en -ahdus / -ähdys expriment des bruits qui se produisent brusquement. Ils sont sans durée et, pour ainsi dire, sans dimension, sans volume. Si d'aventure un mot de ce même type morphologique est au contraire utilisé figurativement, il est alors susceptible d'être employé au partitif:

Vaijan vanhat silmät olivat jotenkin särkyneet, niissä oli $s \ddot{a} i k \ddot{a h} d y s t \ddot{a}$ ja jokin salaileva ajatuskin näytti piilottelevan niissä. (id, $\mathrm{p}$ 231)

"Les vieux yeux du vieillard étaient comme brisés, en eux il y arait de la frayeur et une sorte de pensée dissimulée paraissait s'y cacher."

Le terme säikähdys a, dans l'usage actuel, à peu près perdu sa valeur expressive, tout comme il est advenu de son côté à notre mot français "frayeur".

Ce qui précède a sans doute annoncé déjà assez clairement notre conclusion. Elle est celle-ci: la langue finnoise s'est trouvée dotée de deux procédés pour exprimer le sujet du verbe. L'un consistait à employer le nominatif, l'autre à se servir du partitif. De par ses origines, cette dernière construction ne pouvait avoir lieu qu'en combinaison avec un verbe intransitif et sans accord en nombre entre le sujet et le verbe. Il en 
résulte que le sujet au partitif n'est pas égal au sujet au nominatif. Il est frappé de graves restrictions par rapport à ce dernier. La première est qu'il ne saurait figurer associé à un verbe transitif. Mais cette dernière restriction commence à être ressentie par les locuteurs comme peu tolérable et les grammairiens qui surveillent l'usage présent de la langue littéraire dénoncent assez véhémentement les fautes commises par ceux des usagers qui construisent le sujet partiel (surtout au pluriel) avec un verbe disposant d'un complément d'objet. N'avons-nous pas relevé nous-même la phrase suivante:

Mo n ta Iivanan ja Ofimjankin lasta oli saanut siellä levon. (Elna Pelkonen, op.cit. p. 190)

"Bien des enfants d'Ivan et d'Ophimie y araient trouvé le (dernier) repos."

Cette "faute" est constatée, nous l'avons signalé, surtout dans les parlers orientaux du finnois où on l'a mise au compte de l'influence russe. I.es exemples cités par E. A. Saarimaa (Kielenopas, p 151) ne sauraient toutefois ressortir à cette explication. Ils dénotent seulement que le finnois, tout comme le français, finira par accorder au sujet partiel les mêmes droits qu'au sujet total, c'est-à-dire la faculté de se combiner avec un verbe transitif gourernant un objet.

Ce développement, qui est manifestement en cours. aura naturellement sa répercussion sur l'extension de l'emploi du sujet partiel mais il n'est pas dans notre pouvoir d'anticiper sur l'évolution qui se produira. Constatons seulement que le locuteur de langue finnoise a le loisir de se servir pour l'instant de deux formes de sujet bien que ces deux types n'aipnt pas les mêmes fonctions syntaxiques. Partant de cette dualité, l'usage tend à spécialiser le sujet partiel pour le distinguer du sujet total. Cette spécialisation amène les usagers à déborder du cadre de l'opposition étymologique entre le partiel et le total. D'un cas à l'autre, la distinction stylistique obtenue diffère de nature. Il est rain de vouloir classer ces différenciations selon des catégories logiques plus ou moins nettement définies: partialité et totalité, divisibilité et indivisibilité, défini et indéfini, etc. En gros, le sujet parlant, gardant conscience de la nature traditionnelle de la distinction morphologique, songe 
surtout à l'opposition partiel total mais il l'oublie aisément pour y substituer d'autres distinctions: indétermination, imprécision, emphase, expression de la durée, etc. Nous avons affaire à des développements sémantiques assez analogues à ceux qui ont valeur de leur côté en français à partir de la distinction syntaxique du partitif et du total.

L'intérêt que présentent les faits relevés en finnois est grand du point de vue de la linguistique générale. Ils nous rappellent que les distinctions sémantiques sont issues de différenciations obtenues à partir de différences morphologiques ou syntaxiques. La coexistence de deux ou plusieurs constructions entraîne leur spécialisation respective ou bien l'une des constructions finit par éliminer les autres car l'état de synonymie plus ou moins complète ne dure jamais très longtemps. Le finnois, plus particulièrement le finnois de Finlande, sous sa forme normale, est en train de procéder sous nos yeux à l'exploitation systématique des différenciations qu'il peut développer à partir de la faculté qu'il possède de construire le sujet d'un verbe de trois façons différentes: all nominatif accordé en nombre avec le verbe, au partitif et au nominatif sans accord en nombre avec le verbe. Il est vraisemblable que ces développements prendront dans l'avenir une extension encore plus grande dont les usagers tireront un profit stylistique de plus en plus efficace. 